\title{
DETERMINATION OF VIBRATIONAL EIGENFREQUENCIES OF SEMICONDUCTORS USING TIGHT-BINDING TOTAL ENERGY CALCULATIONS
}

\author{
J. STEPHAN* AND D. SUISKY \\ Institut für Physik, Humboldt-Universität zu Berlin \\ Invalidenstr. 110, 10115 Berlin, Germany
}

We calculate the eigenfrequencies of bulk semiconductors using tight-binding total energy calculations of an ideal and a distorted lattice. We correlate the obtained energy change with the harmonic force constants appearing as parameters in the expansion of the potential energy of the crystal lattice in terms of the displacement. A good agreement of the calculated $q=0$ optical vibrational modes with the experimental values for $\mathrm{Si}, \mathrm{Ge}$, $\mathrm{GaAs}$ and $\mathrm{GaP}$ bulk crystals is obtained. The influence of a surface on the force constants and the vibrational frequencies is studied.

PACS numbers: 63.20.-e

\section{Introduction}

Vibrational eigenfrequencies of bulk semiconductors have been calculated by several theoretical methods. The classical theory of the dynamics of crystal lattices has been developed by Born and Kun Huang [1] and other authors (for a review see [2]). We will demonstrate that another approach starting from tight-binding (TB) total energy calculations can be used for the determination of the vibrational properties as well. In the TB theory the total energy of a sample which is assumed to consist of valence electrons and cores is calculated on the basis of a parametrization of special matrix elements built from binding and anti-binding orbitals of hybridized orbitals and the core-core interaction [3]. Minimizing a total energy functional one obtains the energy for a configuration of cores in fixed positions. Any distortion of this configuration by a vacancy or a surface leads to a change of the total energy.

In the harmonic approximation the difference of the total energy in the equilibrium and the distorted configuration is proportional to the force constants and the displacement. Then, knowing the force constants it is straightforward to obtain the dynamical matrix and to calculate the eigenfrequencies of the considered ideal bulk or distorted sample.

*Present address: Institut für Festkörperphysik, Universität Potsdam, Am Neuen Palais 10, 14415 Potsdam, Germany. 


\section{Theory}

The potential energy $\Phi$ of a system of atoms in dependence on the displacement of atoms $u(m, \kappa)$ reads [1]:

$$
\Phi=\Phi_{0}+\sum_{l, \kappa, i} \Phi_{i}(l, \kappa) u_{i}(l, \kappa)+\sum_{\substack{l, \kappa, i \\ m, \nu, j}} \Phi_{i j}(l \kappa, \dot{m} \nu) u_{i}(l, \kappa) u_{j}(m, \nu)+\ldots
$$

where $l$ and $m$ are the indices of the elementary cell of the lattice, $\kappa$ and $\nu$ indicate the atoms in the elementary cell and $i, j$ label Cartesian coordinates. $\Phi_{i}$ and $\Phi_{i j}$ are the force constants of first and second order. The dynamical matrix $D(\kappa \nu, k)$ is given by

$$
D_{i j}(\kappa \nu, k)=\frac{1}{\sqrt{M_{\kappa} M_{\nu}}} \sum_{m} \Phi_{i j}(0 \kappa, m \nu) \mathrm{e}^{\mathrm{i} k R(m)} .
$$

For the TB calculation a charge self-consistent approach introduced by Neugebauer and other authors $[4,5]$ for the calculation of the configuration of semiconductor interfaces has been used. The total energy of the electron-core system is given by

$$
E=\frac{1}{2} \operatorname{Tr}[P *(F+h)]+\frac{1}{2} \sum_{A} \sum_{B \neq A} \gamma_{A B} Z_{A} Z_{B}
$$

where the first part describes the electronic total energy and the second part - the core-core interaction energy whose calculation was performed with an improved point charge model after Majewski and Vogl [6]. $P$ is the statistical operator, $F-$ the Fock-Hamilton operator and $h$ denotes the one-electron contributions to the total energy. $Z_{A}$ and $Z_{B}$ are the effective charges of the cores and $\gamma_{A B}$ denotes the two-particle matrix elements of the interaction potential between two cores.

\section{Results and discussion}

The results of the calculations are summarized in Table I (bulk frequencies) and Table II (change of the force constants in the presence of a surface). The frequencies have been obtained from the dynamical matrix whose elements depend in cubic crystals on the force constants $\Phi_{x x}$ and $\Phi_{x y}$. The corresponding energy changes per elementary cell for the displacements $u_{x}$ and $u_{x}=u_{y}$ of one atom are $X$ and $Y$, respectively. An example for the dependence of the energy change on the displacement is shown in Fig. 1. One can recognize bulk regions (layer 5 to 19) and surface regions (layer 1 to 4 and 20 to 24). In the bulk region the energy change does not depend on the number of the layer whereas a strong modification of the energy change is observed for the surface region. The latter can be explained by the modified force constants between the atoms.

The theoretical results and the experimental data taken from literature for the bulk materials $\mathrm{C}, \mathrm{Si}, \mathrm{Ge}, \mathrm{GaAs}$ and $\mathrm{GaP}$ are shown in Table I. The frequencies $f_{1}$ and $f_{2}$ can be related to the LO and TO $q=0$ bulk frequencies. The removal of the LO-TO degeneracy depends on the value of $Y$ which is in GaAs and GaP an order of magnitude larger than in $\mathrm{Si}$ and Ge. Moreover, the small values in the homeopolar materials are within the error range of $1 \mathrm{meV}$. The values of $X$ are of the same order of magnitude in both types of materials. For $\mathrm{C}$ both values differ by an order of magnitude from results for $\mathrm{Si}$ and $\mathrm{Ge}$. The comparison of 


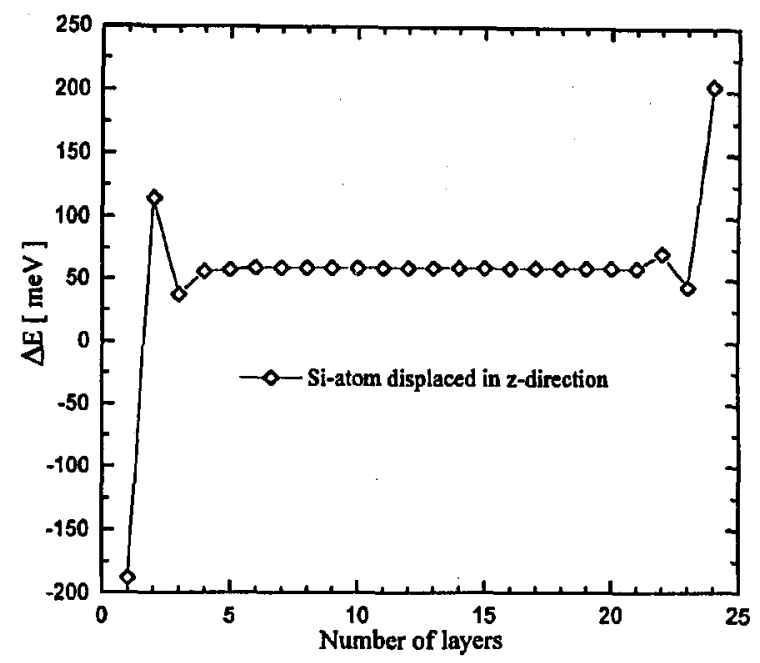

Fig. 1. Change of total energy in dependence on the position of the displaced atom and the direction of displacement. Si $(3 \times 3 \times 24)$ sample. Displacement $\Delta z=0.1 \AA$ perpendicular to the surface. Number of layers in $z$-direction: 24 . Total number of atoms in the sample: 216. Displacement of one atom, all other atoms are fixed at their positions.

TABLE I

Theoretical and experimental values of the optical phonon bulk frequencies. $X$ and $Y$ are given in units of eV/ $\AA^{2}$ and correspond to the force constants $\Phi_{x x}$ and $\Phi_{x y}$, respectively.

\begin{tabular}{c|c|c|c|c|c}
\hline \hline & & $X$ & $Y$ & $f_{1}[\mathrm{THz}]$ & $f_{2}[\mathrm{THz}]$ \\
\hline $\mathrm{C}$ & $\mathrm{TB}$ & 111.482 & -3.795 & 68.88 & 65.39 \\
& {$[7]$} & - & - & $\approx 40$ & $\approx 40$ \\
$\mathrm{Si}$ & $\mathrm{TB}$ & 11.852 & -0.13 & 14.52 & 14.28 \\
& {$[8]$} & - & - & 15.53 & 15.53 \\
$\mathrm{Ge}$ & $\mathrm{TB}$ & 11.32 & -0.122 & 8.83 & 8.68 \\
& {$[9]$} & - & - & 9.12 & 9.12 \\
$\mathrm{GaAs}$ & $\mathrm{TB}$ & 9.236 & -0.658 & 8.21 & 7.36 \\
& $\mathrm{DFT}[10]$ & - & - & - & 8.19 \\
& {$[11]$} & - & - & 8.54 & 8.01 \\
$\mathrm{GaP}$ & $\mathrm{TB}$ & 11.636 & -0.828 & 11.98 & 10.72 \\
& {$[7]$} & - & - & $\approx 11.78$ & $\approx 10.9$
\end{tabular}

the theoretical values with the experimental data show that all theoretical values are below the experimental data (with the exception of $\mathrm{C}$ ). The best agreement between experiment and theory is obtained for GaP. The charge self-consistent procedure for the calculation of the total energy is more appropriate for polar 


\section{TABLE II}

Force constants for $\mathrm{Si}$ in the presence of a surface. $X, Y, Z$, and $A$ are given in units of $\mathrm{eV} / \AA^{2}$ and correspond to the force constants $\Phi_{x x}, \Phi_{x y}, \Phi_{z z}$, and $\Phi_{x z}$, respectively.

\begin{tabular}{c|c|c|c|c}
\hline \hline Layer & $X$ & $Y$ & $Z$ & $A$ \\
\hline 1 & - & - & - & - \\
2 & 2.368 & 4.794 & 24.11 & -3.115 \\
3 & 11.412 & 0.275 & 8.13 & -0.427 \\
4 & 11.534 & 0.020 & 11.38 & -0.07 \\
5 & 11.858 & -0.133 & 11.76 & 0.03 \\
comp. bulk & 11.852 & -0.13 & 11.81 & 0.02
\end{tabular}

materials but it works quite nicely if the eigenfrequencies of the nonpolar materials are of the same order of magnitude. For the stronger bonding in $\mathrm{C}$ the minimizing procedure in terms of a charge redistribution does not seem to be the proper way to proceed and overestimates the frequencies.

The underestimation of the frequencies can be explained by the approximations introduced in our approach where the bulk properties are deduced from the energy changes related to displacements of a limited number of atoms in one elementary cell, despite the fact that the reaction of the whole sample to the disturbance of the equilibrium is taken into account by the charge self-consistent minimization procedure of the total energy. Nevertheless, the good agreement between experimental and theoretical results allows to conclude that the force constants and the frequencies can be obtained from the local disturbance of the equilibrium.

The modification of the force constant under the influence of a surface are shown in Table II for a Si slab consisting of 216 atoms. There are the following modifications in comparison to the bulk region: (i) the relation between the parameters $X$ and $Y$ is strongly modified, (ii) the reduced symmetry in the surface region caused by the nonequivalence of the directions parallel and perpendicular to the surface is described by the additional parameters $Z$ and $A$, (iii) the displacement in the surface region can result in a decrease in the total energy because certain types of displacements simulate the relaxation within the surface region.

The symmetry reduction in the surface region of the investigated Si sample causes a removal of the LO-TO degeneracy due to the nonequivalence of the force constants $\Phi_{x x}$ and $\Phi_{z z}$ as well as $\Phi_{x y}$ and $\Phi_{x z}$ which results in a pronounced deviation of the values of $X$ and $Y$ from the values of $Z$ and $A$, respectively. The force constants are overestimated because the atoms are displaced from their equilibrium positions in the ideal lattice. For an improvement of these parameters given in Table II the relaxation and the reconstruction of the surface region has to be considered. Nevertheless, the results indicate a drastic change of all force constants in the surface region. The influence of an interface on the vibrational properties of samples consisting of different materials can be studied by the same method. 
Summarizing we can state that the method is appropriate for the calculation of the vibrational eigenfrequencies of bulk crystals as well as of crystals containing defects like surfaces and interfaces via the local changes of the force constants taken from tight-binding total energy calculations.

\section{References}

[1] M. Born, K. Huang, Dynamical Theory of Crystal Lattices, Clarendon Press, Oxford 1954.

[2] G.P. Srivastava, The Physics of Phonons, IOP Publishing Ltd., Bristol 1990.

[3] W.A. Harrison, Electronic Structure and the Properties of Solids, Freeman and Company, San Francisco 1980.

[4] J. Neugebauer, R. Enderlein, J. Röseler, Acta. Phys. Pol. A 77, 129 (1990).

[5] L.K. Teles, L.M.R. Scolfaro, A. Josiek, Pessac, R. Enderlein, J.R. Leite, D. Schikora, in: Proc. 23d Int. Conf. on Phys. Semicond., to be published.

[6] J.A. Majewski, P. Vogl, Phys. Rev. B 35, 9666 (1987).

[7] H. Bilz, W. Kress, Phonon Dispersion Relations in Insulators, Springer-Verlag, Berlin 1979.

[8] C.Z. Wang, C.T. Chan, K.M. Ho, Phys. Rev. B 40, 3390 (1989).

[9] M.T. Yin, M.L. Cohen, Phys. Rev. B 26, 3259 (1982).

[10] G. Schwarz, private communication.

[11] K. Kunc, R.M. Martin, Phys. Rev. B 24, 231 (1981). 